\title{
High-Sensitivity Absorption Spectroscopy in Fe II
}

\author{
Scott D. Bergeson \\ scott.bergeson@byu.edu \\ K. L. Mullman \\ J. E. Lawler
}

Follow this and additional works at: https://scholarsarchive.byu.edu/facpub

Part of the Astrophysics and Astronomy Commons

\section{Original Publication Citation}

S. D. Bergeson, K. L. Mullman, and J. E. Lawler. High-sensitivity absorption spectroscopy in Fe II. Astrophys. J. 464 (2), 1050-1053 (1996).

\section{BYU ScholarsArchive Citation}

Bergeson, Scott D.; Mullman, K. L.; and Lawler, J. E., "High-Sensitivity Absorption Spectroscopy in Fe II" (1996). Faculty Publications. 1829.

https://scholarsarchive.byu.edu/facpub/1829

This Peer-Reviewed Article is brought to you for free and open access by BYU ScholarsArchive. It has been accepted for inclusion in Faculty Publications by an authorized administrator of BYU ScholarsArchive. For more information, please contact ellen_amatangelo@byu.edu. 


\title{
HIGH-SENSITIVITY ABSORPTION SPECTROSCOPY IN Fe II
}

\author{
S. D. Bergeson, K. L. Mullman, and J. E. Lawler \\ Physics Department, University of Wisconsin-Madison, Madison, WI 53706 \\ Received 1995 October 25 ; accepted 1996 January 5
}

\begin{abstract}
We report the first measurements of UV oscillator strengths ( $f$-values) in $\mathrm{Fe}$ II from a high-sensitivity absorption experiment developed at the University of Wisconsin. The accuracy of our measurements is demonstrated by our reproducing well-known $f$-value ratios in $\mathrm{Fe}$ I and $\mathrm{Fe}$ II. The first laboratory $f$-value measurement of the $160.845 \mathrm{~nm}$ transition in $\mathrm{Fe}$ II is presented and compared to values in the literature. While this paper focuses on Fe II, the high-sensitivity absorption method that we have developed is applicable to essentially every element in the periodic table, for both neutral and singly ionized species, over a broad range of wavelength and line strength.
\end{abstract}

Subject headings: atomic data - ISM: abundances - methods: laboratory - ultraviolet: general

\section{INTRODUCTION}

There is a need for improved oscillator strengths $(f$ values) of many VUV transitions of iron group atomic ions. These data are needed for interpreting high-quality absorption spectra from the Goddard High Resolution Spectrograph on the Hubble Space Telescope and spectra from other orbiting observatories (Leckrone et al. 1993).

In this paper we describe a new high-sensitivity absorption experiment for measuring $f$-values of VUV transitions. We report measurements of UV $f$-value ratios for wellknown $\mathrm{Fe}_{\mathrm{I}}$ and $\mathrm{Fe}$ II line pairs. These measurements demonstrate the reliability of our method. Extensions in the dynamic range are discussed. We report the first laboratory measurement of the $\mathrm{Fe}$ II $160.845 \mathrm{~nm} f$-value and compare to values in the literature. This VUV line is of particular interest as one of a set of UV and VUV Fe II resonance lines spanning a large range of $f$-values. This set of increasingly accurate $f$-values makes it possible to study the Fe II abundance in the interstellar medium with a large dynamic range in column density (Cardelli \& Savage 1995). For example, the weakest lines would be used to determine an accurate column density along a line of sight for which the core of a strong line is highly saturated. Stronger lines are used to study high-velocity component structure along this line of sight (Cardelli \& Savage 1995). While this discussion focuses on $\mathrm{Fe}^{+}$, the high-sensitivity absorption method is applicable to essentially every element in the periodic table, for both neutral and singly ionized species, over a wide range in wavelength and line strength.

Absorption spectroscopy has long been widely used to determine $f$-values, but usually at longer wavelengths and usually on neutral atoms. For example, the most accurate relative $\mathrm{Fe}$ i $f$-values were measured by the Oxford group (Blackwell et al. 1979) using the absorption method on a sample of $\mathrm{Fe}$ vapor in an oven at local thermodynamic equilibrium (LTE). Radiative lifetimes measured using timeresolved laser-induced fluorescence (LIF) have established an absolute scale for the Oxford $f$-values which is accurate to within a few percent (O'Brian et al. 1991 and references therein). LIF experiments are now operating in the VUV on both neutral atoms and atomic ions (O'Brian \& Lawler 1991; Bergeson \& Lawler 1993; Zerne, Larsson, \& Svanberg 1994). Clearly new experiments are needed to determine relative absorption $f$-values and/or emission branching fractions in the VUV in order to exploit the life- time data and determine individual $f$-values. Emission branching fractions measured using Fourier transform (FT) spectroscopy have greatly expanded the $f$-value database for Fe I lines (O'Brian et al. 1991) and Fe II (Bergeson et al. 1996). A particularly attractive possibility is to combine emission branching fractions with relative absorption $f$ values using the "bow tie" least-squares adjustment technique (Cardon, Smith, \& Whaling 1979). This redundant approach is desirable in view of the experimental difficulties of establishing an accurate radiometric scale when measuring emission branching fractions in the VUV.

Our new absorption experiment uses a hollow cathode glow discharge to establish a gas-phase sample of $\mathrm{Fe}^{+}$. This sample is not in LTE, and thus we are limited to measuring relative $f$-values from common lower levels. This limitation is not serious because many low-lying $\mathrm{Fe}^{+}$levels are connected to reference lines above $200 \mathrm{~nm}$ with accurately known $f$-values (Bergeson et al. 1996). The lack of LTE is advantageous because the experiment can probe chemical elements, ionization states, and lower level energies which are not accessible using ovens. Our experiment uses an electron storage ring to provide a smooth continuum covering the entire VUV with a very high spectral radiance and uses a $3 \mathrm{~m}$ focal length vacuum echelle spectrometer to provide resolving powers up to 350,000 . This experiment achieves sensitivity to very small fractional absorptions using a VUV-sensitive CCD array. Enormous gains in sensitivity are achieved using detector arrays in absorption spectroscopy (Wamsley, Mitsuhashi, \& Lawler 1993; Childs et al. 1994).

\section{HIGH-SENSITIVITY ABSORPTION}

Some ideas underlying our experiment are described in a previous publication (Lawler, Bergeson, \& Wamsley 1993). We measure relative absorption $f$-values for transitions originating from a common lower level. A schematic diagram of the experiment is shown in Figure 1. As in most classic absorption measurements, three major components comprise our experiment: a continuum source, a lowpressure gas-phase sample of absorbing atoms or ions, and a spectrometer/detector system.

Our continuum source is the white light beam line on the Aladdin Storage Ring at the Synchrotron Radiation Center. Typical operating parameters for this electron storage ring are $800 \mathrm{MeV}$ beam energy, $200 \mathrm{~mA}$ current, and $2.083 \mathrm{~m}$ 


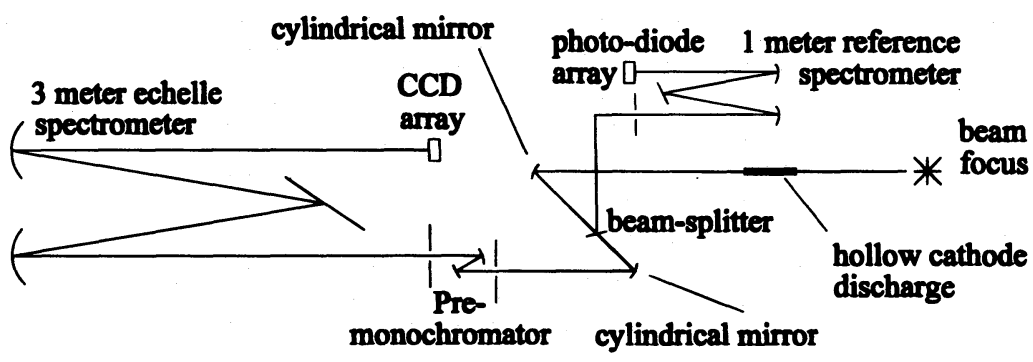

Fig. 1.-Schematic diagram of the high-sensitivity absorption experiment

magnetic bending radius. The continuum is both spectrally smooth and very stable, covers the entire VUV range, and has a spectral radiance greater than $10^{3}$ times that of an arc lamp. Our low-pressure gas-phase sample of $\mathrm{Fe}$ and $\mathrm{Fe}^{+}$is a $1 \mathrm{~cm}$ inner diameter, $10 \mathrm{~cm}$ length, water-cooled hollow cathode discharge (HCD). Described in detail elsewhere (Bergeson, Mullman, \& Lawler 1994), this source produces useful populations of atoms and ions distributed throughout many metastable levels. At Ar buffer gas pressures of 1 to 2 torr and at currents ranging from 0.02 to $1.5 \mathrm{amps}$, we measure $\mathrm{Fe}^{+}$ground level number densities from $10^{8} \mathrm{~cm}^{-3}$ to $10^{11} \mathrm{~cm}^{-3}$.

Because we are measuring relative $f$-values, we use two spectrometer/detector systems. One is used to measure a "reference" line. The other is used to measure the "unknown" line. This method eliminates the need to measure the reference and unknown lines alternatively, reducing data acquisition time, and relaxing requirements for long-term stability in the hollow-cathode source.

One of the spectrometer/detector systems is an Acton Research Corporation model VM-510, $1 \mathrm{~m}$ vacuumcompatible spectrometer with a Princeton Instruments 15.5 bit 1024 element photodiode array (PDA). The resolving power of this system is around $2 \times 10^{4}$, limited by the $25 \mu \mathrm{m}$ spacing of diodes in the array. This lower resolving power system is used to monitor the carefully selected, wellisolated reference line. The other spectrometer/detector system is a $3 \mathrm{~m}$ focal length vacuum compatible echelle spectrograph equipped with a VUV-sensitive CCD detector array. The practical resolving power of this instrument equipped with this detector, when used with the $256 \mathrm{~mm}$ wide, $63^{\circ}$ blaze, echelle grating is $\sim 3 \times 10^{5}$. The CCD is a Scientific Imaging Technologies back-thinned deep UVsensitive device, in a camera head from Princeton Instruments. The CCD format is square, 512 pixels on a side. Because the spectrometer operates from 21st to 36th order for these measurements, we use a McPherson model 234/303 Seya monochromator as an order-sorter. We limit the Seya bandpass to $0.1 \mathrm{~nm}$, reducing stray light in the high-resolution spectrometer.

The light beam from the storage ring is coupled into the 3 $\mathrm{m}$ echelle spectrometer using two off-axis cylindrical mirrors that reshape the beam to match the slit size and angular acceptance of the spectrometer. A similar coupling system sends light into the reference spectrometer by reshaping a $\sim 4 \%$ reflected beam from an uncoated $\mathrm{MgF}_{2}$ beam splitter. This coupling system uses two off-axis cylindrical mirrors and a cylindrical lens. The lower resolving power and higher etendue of the reference spectrometer results in higher light fluxes on the detector. This enables us to use a diode array instead of a more sensitive CCD array.
A sample absorption spectrum of the Fe II transition at $258.588 \mathrm{~nm}$ is shown in Figure 2. The rms fractional noise on the continuum is 0.003 , which is consistent with Poisson statistics in the photoelectrons in the CCD array. This multichannel absorption experiment is superior to singlechannel sequentially scanned absorption experiments. In a single-channel experiment, fluctuations or flicker in the continuum source are mapped into noise, directly limiting the sensitivity of the measurements. In a multichannel experiment such as ours, all spectral elements are measured simultaneously, making intensity fluctuations or flicker inconsequential. The sensitivity of the measurements is now set by Poisson statistics of the photoelectrons, which accumulate quickly in multichannel detection. Childs et al. (1994) have achieved sensitivities to fractional absorptions of 0.00001 in a relatively simple experiment using a detector array.

We discriminate against line emission from the HCD with a digital subtraction technique (Wamsley et al. 1993). A spectrum of the synchrotron radiation after it traverses the HCD is a combination of the synchrotron continuum radiation, absorption features from atoms and ions in the line of sight, and line emission from the HCD. By blocking the synchrotron radiation, we can measure the line emission from the HCD and subtract it from the first spectrum and thereby observe only the continuum radiation with absorption features. Pixel-to-pixel variations in the quantum efficiency of the array can be divided out using a dark signal-corrected, high signal-to-noise ratio spectrum of the synchrotron radiation when the HCD is turned off.

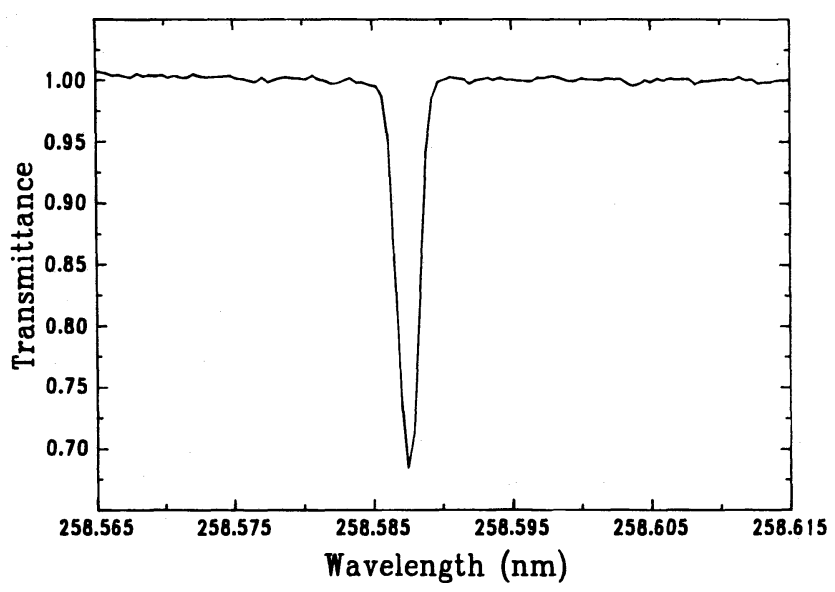

Fig. 2.-Sample absorption spectrum of the Fe II transition at 258.588 $\mathrm{nm}$. The fractional noise on the continuum in 0.003 and is consistent with Poisson statistics in the photoelectrons in the CCD array. 
We have paid particular attention to minimizing stray light in our experiment, and we correct for it in our measurements. The stray light level inside the spectrometer system is determined by measuring the photon flux between orders of the $3 \mathrm{~m}$ spectrometer. After each absorption measurement, we leave the Seya bandpass at $0.1 \mathrm{~nm}$ and tune the $3 \mathrm{~m}$ spectrometer $0.3-0.5 \mathrm{~nm}$ from the central wavelength of the Seya bandpass and measure this "interorder" photon flux. This interorder photon flux is typically $1 \%$ of the continuum flux at the peak of the Seya bandpass. In addition, we directly measure the stray light level during an absorption experiment. We place a $\mathrm{Hg}$ vapor cell in the line of sight and measure absorption at the $254 \mathrm{~nm}$ resonance line. When the column density is high; the observed absorption feature is completely saturated. Measuring the light level at line center under these conditions provides an independent and more direct measure of the stray light level which agrees with our interorder measurements.

The linearity of the detector arrays is an important issue when measuring weak absorption features because it affects the accuracy of the digital subtraction. This is especially important when the peak of the line emission from the HCD is comparable to the continuum emission from an arc lamp. Recently, Meningen et al. (1995) have studied the effects of nonlinearity in high-sensitivity absorption measurements with a PDA. They found a nonlinearity of roughly 0.01 across the full-well of each PDA pixel. Digital signal processing can be used to correct such nonlinearities. We measure the nonlinearity of our CCD array and find it to be less than 0.001 from $4 \%$ full-well to $85 \%$ full-well. When the line emission from the HCD is much less than the synchrotron continuum, which is true in our system because of careful design of the optical coupling, these very small nonlinearities have no influence on the measured absorption line.

At resolving powers of $\sim 3 \times 10^{5}$, we cannot directly observe the spectral shape of lines emitted from the HCD. In our curve-of-growth analysis, we assume that the spectral line shape is a full Voigt profile. The Lorentzian contribution to the Voigt profile is assumed to be primarily radiative (natural) broadening. The temperature of the atoms in the HCD as a function of input power is found by finding the best overall consistency in absorption measurements made over a wide range of HCD input powers. For the data reported here, all of the measurements are made on or near the linear part of the curve of growth, minimizing the influence of uncertainties in the spectral line shape. We see some evidence for a non-Maxwellian velocity distribution of the ions in our discharge. A model of our HCD suggests this may be due to competition between collisional thermalization rates that "fill out" the Maxwellian velocity distribution and ion loss rates (ambipolar diffusion). Because the energetic ions escape more rapidly, the tail of the Maxwellian velocity distribution is underdeveloped, resulting in a truncated Voigt profile. This process is often described as "diffusive cooling." We are currently working on the curve of growth in order to expand the dynamic range of the experiment.

The experiment is now sensitive to fractional absorptions of 0.0003 at intermediate resolving powers and of 0.003 at high resolving powers. The sensitivity will be improved by using better VUV optics and a more advanced detector array, and possibly by moving the experiment to a storage ring beam line with an insertion device (wiggler or undulator). Sensitivity to fractional absorptions as small as 0.0001 at high resolving powers and at wavelengths as short as $120 \mathrm{~nm}$ should be achievable in the future. This sensitivity goal corresponds to measuring equivalent widths as small as $0.00003 \mathrm{~cm}^{-1}$ or ionic column densities of $\mathrm{Nl}=10^{7} \mathrm{~cm}^{-2} / f$.

\section{RESULTS AND DISCUSSION}

Table 1 compares results of our absorption measurements in $\mathrm{Fe} \mathrm{I}$ and $\mathrm{Fe}$ II with values from the literature. The number in parentheses following an entry in the table is the uncertainty in the last digits of the entry. Blackwell et al. (1979), measured relative oscillator strengths in $\mathrm{Fe} I$ with a $1 \sigma$ uncertainty of $0.5 \%$. The work of Bergeson et al. (1996) combines carefully measured branching fractions measured using Fourier transform spectroscopy with high-accuracy radiative lifetimes from the recent literature (Biemont et al. 1991) to determine $f$-values accurate to better than $5 \%$ for the strong lines listed in Table 1 . The values in Table 1 from the NBS critical compilation (Fuhr, Martin, \& Wiese 1988) are renormalized using high-accuracy radiative lifetimes to provide the best overall absolute scale for their listed branching fractions. Error bars on ratios from Fuhr, Martin, \& Wiese (1988) are decreased to $\pm 10 \%$ to reflect the improved normalization provided by new lifetime measurements. At this point, we consider the work of Blackwell et al. (1979) and of Bergeson et al. (1996) to be more accurate than the results of this experiment. The results in Table 1 illustrate that we can reliably measure relative absorption

TABLE 1

Comparison of Absorption $f$-Value Ratios Measured in the High-Sensitivity Absorption Experiment with Values IN THe Literature

\begin{tabular}{|c|c|c|c|c|c|c|c|c|c|c|c|c|c|}
\hline \multirow[b]{2}{*}{ ElEMENT } & \multicolumn{3}{|c|}{ LOWER LEVEL } & \multicolumn{4}{|c|}{ UPPER LEVEL 1} & \multicolumn{4}{|c|}{ UPPER LEVEL 2} & \multicolumn{2}{|r|}{$f_{1} / f_{2}$} \\
\hline & $\begin{array}{l}\text { Energy } \\
\left(\mathrm{cm}^{-2}\right)\end{array}$ & Term & $J$ & $\begin{array}{c}\lambda_{1} \text { in Air } \\
(\mathrm{nm})\end{array}$ & $\begin{array}{l}\text { Energy } \\
\left(\mathrm{cm}^{-1}\right)\end{array}$ & Term & $J$ & $\begin{array}{c}\lambda_{2} \text { in Air } \\
(\mathrm{nm})\end{array}$ & $\begin{array}{l}\text { Energy } \\
\left(\mathrm{cm}^{-1}\right)\end{array}$ & Term & $J$ & This Work & Other Values \\
\hline $\mathrm{Fe}_{\mathrm{I}}$ & 0.000 & $a^{5} D$ & 4 & 296.690 & 33695.394 & $y^{5} F^{o}$ & 5 & 298.357 & 33507.120 & $y^{5} D^{o}$ & 3 & $1.49(9)$ & $1.510(8)^{\mathrm{a}}$ \\
\hline Fe I. & 888.129 & $a^{5} D$ & 1 & 295.737 & 34962.144 & $y^{5} F^{o}$ & 1 & 302.403 & 33946.929 & $z^{3} P^{o}$ & 2 & $2.0(3)$ & $2.084(10)^{\mathrm{a}}$ \\
\hline Fe II...... & 0.000 & $a^{6} D$ & $9 / 2$ & 238.204 & 41968.046 & $z^{6} F^{o}$ & $11 / 2$ & 259.940 & 38458.981 & $z^{6} D^{o}$ & $9 / 2$ & $1.27(8)$ & $1.34(3),{ }^{\mathrm{b}} 1.35(14)^{\mathrm{c}}$ \\
\hline $\mathrm{Fe}$ II ...... & 0.000 & $a^{6} D$ & $9 / 2$ & 259.940 & 38458.981 & $z^{6} D^{o}$ & $9 / 2$ & 258.588 & 38660.043 & $z^{6} D^{o}$ & $7 / 2$ & $3.55(21)$ & $3.47(13),{ }^{b} 3.37(35)^{c}$ \\
\hline Fe II...... & 0.000 & $a^{6} D$ & $9 / 2$ & 259.940 & 38458.981 & $z^{6} D^{o}$ & $9 / 2$ & 234.350 & 42658.224 & $z^{6} P^{o}$ & $7 / 2$ & $2.00(14)$ & $2.10(5),{ }^{\mathrm{b}} 2.12(21)^{\mathrm{c}}$ \\
\hline Fe II...... & 0.000 & $a^{6} D$ & $9 / 2$ & 234.350 & 42658.224 & $z^{6} P^{o}$ & $7 / 2$ & 258.588 & 38660.043 & $z^{6} D^{o}$ & $7 / 2$ & $1.56(12)$ & $1.65(6),{ }^{\mathrm{b}} 1.59(16)^{\mathrm{c}}$ \\
\hline $\mathrm{Fe}$ II....... & 7955.299 & $a^{4} D$ & $7 / 2$ & 275.574 & 44232.512 & $z^{4} F^{o}$ & $9 / 2$ & 273.955 & 44446.878 & $z^{4} D^{o}$ & $7 / 2$ & $1.22(8)$ & $1.19(8){ }^{\mathrm{d}} 1.19(12)^{\mathrm{c}}$ \\
\hline
\end{tabular}

a Blackwell et al. 1979.

b Bergeson et al. 1996.

c Values listed in Fuhr, Martin, \& Wiese 1988 rescaled with accurate lifetime from Biemont et al. 1991.

d Bergeson, Mullman, \& Lawler 1994. 
TABLE 2

Fe II ABSORPTION $f$-VALUE FOR $\lambda_{\text {yac }}=160.845 \mathrm{~nm}$ TRANSITION $a^{6} D_{9 / 2}\left(0.000 \mathrm{~cm}^{-1}\right)-y^{6} P_{7 / 2}^{o}\left(62171.615 \mathrm{~cm}^{-1}\right)$

\begin{tabular}{ll}
\hline \multicolumn{1}{c}{ Source } & \multicolumn{1}{c}{$f$-Value } \\
\hline This work $\ldots \ldots \ldots \ldots \ldots \ldots \ldots \ldots \ldots \ldots \ldots \ldots \ldots \ldots \ldots$ & $0.058(5)$ \\
Cardelli \& Savage $1995 \ldots \ldots \ldots \ldots \ldots \ldots \ldots \ldots \ldots$ & $0.0619(62)$ \\
Shull, Van Steenberg, \& Seab $1983 \ldots \ldots \ldots \ldots \ldots \ldots$ & $0.062(18)$ \\
Nussbaumer, Pittini, \& Storey $1981 \ldots \ldots \ldots \ldots \ldots$ & 0.0963 \\
Kurucz 1988 $\ldots \ldots \ldots \ldots \ldots \ldots \ldots \ldots \ldots \ldots \ldots \ldots \ldots$ & 0.0716 \\
\hline
\end{tabular}

$f$-value ratios ranging up to about 4 . With an improved curve of growth we anticipate a significantly larger dynamic range.

With this new high-sensitivity absorption experiment, we measure the absorption $f$-value of the Fe II $258.588 \mathrm{~nm}$ transition relative to the $160.845 \mathrm{~nm}$ transition to be 1.20 (9). The absolute value of the $258.588 \mathrm{~nm} f$-value is $0.0690(25)$ (Bergeson et al. 1996), making the absolute $f$-value of the $160.845 \mathrm{~nm}$ transition $0.058(5)$. This first laboratory measurement of the $160.845 \mathrm{~nm} f$-value is compared with values from the literature in Table 2.

\section{SUMMARY}

We have developed a new experiment for making highsensitivity absorption measurements in $\mathrm{Fe}$ II and in the spectra of other ions and atoms. The fidelity of the experiment is demonstrated by our reproducing the highly accurate $\mathrm{Fe}_{\mathrm{I}} f$-values of Blackwell et al. (1979) as well as well-known $f$-values in $\mathrm{Fe}$ II (Bergeson et al. 1996). The experiment is now being used to measure $f$-values of $\mathrm{Fe}$ II VUV line by comparing to lines above $200 \mathrm{~nm}$ with known $f$-values. This experiment will also be useful in generating "bow ties" (Cardon et al. 1979) for least-squares adjustment of $f$-values based on a combination of emission branching ratios and absorption measurements. While the presentation here centers on $f$-value measurements in $\mathrm{Fe}$ II, this method is applicable to essentially every element in the periodic table, for both neutral and singly ionized species, over a wide range of wavelength and line strength.

This research is supported by NASA under grant NAGW-2908, and by the National Science Foundation under grant DMR-9212658 to the Synchrotron Radiation Center. We thank Mr. M. Sakai for assistance during the final stages of data collection.

\section{REFERENCES}

Bergeson, S. D., \& Lawler, J. E. 1993, ApJ, 414, L137

Bergeson, S. D., Mullman, K. L., \& Lawler, J. E. 1994, ApJ, 435, L157

Bergeson, S. D., Mullman, K. L., Wickliffe, M. E., Lawler, J. E., Litzen, U., \& Johansson, S. 1996, ApJ, 464, 1044

Biemont, E., Baudoux, M., Kurucz, R. L., Ansbacher, W., \& Pinnington, E. H. 1991, A\&A, 249, 539

Blackwell, D. E., Ibbetson, P. A., Petford, A. D., \& Shallis, M. J. 1979, MNRAS, 186, 633

Cardelli, J. A., \& Savage, B. D. 1995, ApJ, 452, 275

Cardon, B. L., Smith, P. L., \& Whaling, W. 1979, Phys. Rev., A20, 2411

Childs, M. A., Menningen, K. L., Toyoda, H., Ueda, Y., Anderson, L. W., \& Lawler, J. E. 1994, Phys. Lett. A, 194, 119

Fuhr, J. R., Martin, G. A., \& Wiese, W. L. 1988, J. Phys. Chem. Ref. Data, 17, Suppl. 4, 108
Kurucz, R. L. 1988, Trans. IAU, 28, 168

Lawler, J. E., Bergeson, S. D., \& Wamsley, R. C. 1993, Phys. Scripta, T47, 29

Leckrone, D. S., Johansson, S., Wahlgren, G. M., \& Adelman, S. J. 1993, Phys. Scripta, T47, 149

Menningen, K. L., Childs, M. A., Toyoda, H., Ueda, Y., Anderson, L. W., \& Lawler, J. E. 1995, Contr. Plasma Phys., 35, 359

Nussbaumer, H., Pittini, M., \& Storey, P. J. 1981, A\&A, 102, 351

O’Brian, T. R., \& Lawler, J. E. 1991, Phys. Rev., A44, 7134

O'Brian, T. R., Wickliffe, M. E., Lawler, J. E., Whaling, W., \& Brault, J. W. 1991, J. Opt. Soc. Am. B8, 1185

Shull, J. M., Van Steenberg, M., \& Seab, C. G. 1983, ApJ, 271, 408

Wamsley, R. C., Mitsuhashi, K., \& Lawler, J. E. 1993, Rev. Sci. Instr., 64, 45 Zerne, R., Larsson, J., \& Svanberg, S. 1994, Phys. Rev., A49, 128 